\title{
Synaptic Actions of Identified Peptidergic Neuron R15 in Aplysia. III. Activation of the Large Hermaphroditic Duct
}

\author{
A. Alevizos, ${ }^{1}$ K. R. Weiss, ${ }^{2, a}$ and J. Koester ${ }^{2}$ \\ Center for Neurobiology and Behavior, 'Department of Physiology and Cellular Biophysics and 2Department of Psychiatry, \\ College of Physicians and Surgeons, Columbia University, New York, New York 10032
}

\begin{abstract}
The purpose of the study described in this and the preceding two companion papers was to determine the synaptic actions of neuron R15, an endogenously bursting neurosecretory cell in Aplysia, as a step toward determining its physiological function. The results described in this paper demonstrate that activity in R15 activates anterograde peristaltic movements in the segment of the large hermaphroditic duct through which eggs move during egg-laying behavior. This action is mimicked by $R 15 \alpha 1$ peptide, a putative transmitter of R15. The neuroendocrine bag cells, which initiate egg laying when they fire in a population burst, have been shown by others to excite R15. Our data suggest that R15 mediates excitatory effects of the bag cells on the large hermaphroditic duct. Taken with the results of the two companion papers, these data support the hypothesis that R15 integrates various aspects of egg-laying behavior. The desensitization of R15's postsynaptic actions may complement the long-lasting refractoriness of the bag cells described by others, with both effects contributing to the episodic nature of egg laying.
\end{abstract}

The goal of this study was to determine the synaptic actions of $\mathrm{R} 15$, a peptidergic bursting neuron located in the abdominal ganglion of Aplysia. In our initial studies, we focused on the central synaptic actions of R15 that may be involved in egglaying behavior. Egg laying is initiated by a population burst in the neuroendocrine bag cells (Pinsker and Dudek, 1977). One effect of the bag-cell burst in vitro is excitation of $\mathrm{R} 15$ (Branton et al., 1978). This excitation is relayed by $R 15$ central synapses to the R25/L25 network, which drives respiratory pumping, and to motoneuron $\mathrm{L} 7$, which causes connective contraction (Alevizos et al., 1991a,b).

R15 sends numerous processes to the periphery, so we reasoned that R15 might also exert peripheral synaptic actions. Although Rittenhouse and Price (1985) recorded spike activity from axonal branches of R15 in a variety of visceral organs, they observed no peripheral physiological effects of $R 15$, perhaps because the peripheral actions of R15 exhibit properties

\footnotetext{
Received Apr. 13, 1990; revised Dec. 10, 1990; accepted Dec. 13, 1990.

We thank M. Skelton for critically reviewing an earlier draft of this paper. This work was supported by NIH Grants NS14385 and GM32099, National Institute of Mental Health Grant MH36730, an NIH BRSG grant, and the Klosk Foundation.

Correspondence should be addressed to Dr. J. Koester, Center for Neurobiology and Behavior, The New York State Psychiatric Institute, 722 West 168 Street New York, NY 10032.

a Present address: Mount Sinai School of Medicine, New York, NY 10029. Copyright (c) 1991 Society for Neuroscience $0270-6474 / 91 / 111282-09 \$ 03.00 / 0$
}

similar to its central actions; that is, they decrease rapidly with maintained firing of R15 (Alevizos et al., 1991a,b). Indeed, by blocking spontaneous activity in R 15 for $2 \mathrm{hr}$ before testing, we found that activity in R15 elicits peristaltic activity of the large hermaphroditic duct in vitro.

The population burst in the bag cells has a direct effect on ovulation. The egg-laying hormone (ELH) released during the burst acts on the ovotestis to release mature oocytes (Coggeshall, 1970; Arch, 1976; Rothman et al., 1983). The free oocytes then enter the small hermaphroditic duct and are propelled by cilia to the accessory genital mass (Coggeshall, 1970). There, the mature oocytes are mixed with secretions of the albumen gland and fertilized by sperm that the animal received previously during copulation. The fertilized eggs are coated with mucus before leaving the accessory genital mass via the large hermaphroditic duct (LHD). Final secretions are added as the eggs travel through the red hemiduct of the LHD. By this time, the eggs have been covered by a gelatinous coat and formed into a continuous egg cordon, which can reach up to $17.5 \mathrm{~m}$ in length (Kandel, 1976). The secretions of the red hemiduct, which are adhesive, presumably facilitate the attachment of the egg cordon to the substrate after oviposition (Thompson and Bebbington, 1969; Coggeshall, 1970). The passage of the entire egg cordon through the red hemiduct typically takes from $30-90 \mathrm{~min}$ (Thompson and Bebbington, 1969; Coggeshall, 1972; Ferguson et al., 1989). From the red hemiduct, the eggs enter the genital groove in the external body wall, through which they move anteriorly to the head, where they are deposited on the substrate.

Data presented in this paper provide further support for the hypothesis that R15 may control various aspects of egg-laying behavior. We found that $\mathrm{R} 15$ enhances anterograde peristaltic activity of the red hemiduct, the portion of the LHD that transports the egg cordon to the genital groove.

Some of these results have been described in a preliminary communication (Alevizos et al., 1989b).

\section{Materials and Methods}

Morphology. To trace the projection of R15 processes to the periphery, the cell body was filled with fluorescent dyes. Lucifer yellow ( $5 \%$ aqueous solution; Sigma) or 5,6-carboxyfluorescein (Kodak; 3\% aqueous solution; Rao et al., 1986) was injected into the soma of $R 15$ by iontophoresis (10-nA pulses of inward current, $50 \%$ duty cycle for 12-14 hr or by pressure injection (8-12 psi). To minimize the distance required for diffusion of the dye, small animals $(3-8 \mathrm{gm}$ ) were used for these experiments. Following injection, the dye was allowed to diffuse while the preparation was kept at $15^{\circ} \mathrm{C}$ for $48 \mathrm{hr}$ in modified L15 culture medium, which is described by Alevizos et al. (1991a). When 5,6-carboxyfluorescein was used for tracing, $5 \mathrm{~mm}$ probenecid (Sigma) was added to the bathing medium to reduce transport of the dye out of the cell (DiVirgilio et al., 1988). For cells injected with Lucifer yellow, the 
preparation was fixed for $2 \mathrm{hr}$ in a solution of $0.1 \mathrm{M}$ phosphate buffer (pH, 7.6) containing 4\% paraformaldehyde plus $30 \%$ sucrose. The fixed tissue was washed with $0.1 \mathrm{M}$ phosphate buffer and cleared with a phosphate buffer solution containing glycerol $(1: 6)$. The ganglion was then viewed and photographed with a fluorescence microscope.

In vitro recording techniques. Standard electrophysiological techniques were used, as described by Alevizos et al. (1991a). As explained in that paper, a "nondesensitizing washing protocol" was employed to prevent the desensitizing effects of the synaptic actions of R 15 when it fires spontaneously: the ganglion was placed in a bath containing high$\mathrm{Mg}^{2+} / \mathrm{low}-\mathrm{Ca}^{2+}$ saline to prevent transmitter release; before the preparation was washed with standard saline [artificial seawater (ASW)], R15 was impaled and prevented from firing by hyperpolarizing current injection. It then was kept hyperpolarized for $2 \mathrm{hr}$ before it was released and allowed to burst spontaneously, at which time its postsynaptic actions were observed.

The reduced preparation most used in this study to examine the synaptic actions of $R 15$ consisted of the large hermaphroditic duct connected to the isolated abdominal ganglion by the genital nerve. The genital artery, which perfuses the LHD, was cannulated with a polyethylene tube and perfused continuously at $1-3 \mathrm{ml} / \mathrm{min}$ with ASW. In a few experiments, the connections of the abdominal ganglion to the head ganglia by the pleuroabdominal connectives were maintained (Alevizos et al., 1991a).

Contractions of the large hermaphroditic duct (LHD) or its white hemiduct or red hemiduct were recorded in vitro by means of an isotonic displacement transducer (Harvard Apparatus, model 52-9511) attached to the duct by a silk ligature or a hooked insect pin. For quantification of the time integral of the contractions, the arca cnclosed by the contraction waveform recorded on a Gould 2400 chart recorder was measured by hand. This envelope of the contractions that was measured included both tonic and phasic movements.

To determine if connections from R15 in the abdominal ganglion to the muscle of the hermaphroditic duct are mediated by central polysynaptic pathways, the abdominal ganglion was placed in a 1-ml well isolated with Vaseline from the rest of the recording chamber. The bathing medium of the well was replaced with either a high-divalentcation solution containing $3 \times$ normal $\mathrm{Mg}^{2+}$ and $3 \times$ normal Ca ${ }^{2+}$ (Alevizos et al., 1991a) to block polysynaptic pathways (including electrically coupled cells), or with a solution containing $10 \mathrm{mM} \mathrm{Co}{ }^{2+}, 0 \mathrm{mM} \mathrm{Ca}^{2+}$, $460 \mathrm{~mm} \mathrm{NaCl}, 55 \mathrm{~mm} \mathrm{MgCl}_{2}$, and $10 \mathrm{mM} \mathrm{KCl}$ (adjusted to $\mathrm{pH} 7.6-7.8$ with HEPES buffer, $10 \mathrm{~mm}$ ), which completely blocks chemical synaptic transmission. (The normal concentrations of $\mathrm{Ca}^{2+}$ and $\mathrm{Mg}^{2+}$ in Aplysia saline are 10 and $55 \mathrm{~mm}$, respectively.) Isolating the LHD and peripheral nerves from the test solution bathing the ganglion prevented the highdivalent-cation solution from blocking axonal conduction along the axons that cells in the ganglion send to the periphery. It also prevented the low- $\mathrm{Ca}^{2+}$ solution from blocking synaptic transmission in the periphery.

In vivo recording techniques. The movements of the LHD during induced egg laying were monitored in vivo by measuring impedance changes in animals weighing $200-300 \mathrm{gm}$. The anesthetized animal was opened by a small incision in the body wall, and the bare end of an insulated, multistranded platinum-iridium wire (Medwire; Mt. Vernon, NY) was inserted into the red hemiduct of the LHD. Another wire was looped around the LHD close to the site of insertion of the first wire. The opposite ends of the two wires were passed through the body wall and connected to an impedance converter (model 2991, Biocom Inc.; Culver City, CA) during the recording sessions. The incision was closed with a double layer of silk sutures, and subjects were allowed $36-48 \mathrm{hr}$ to recover before recording began. Quantification of the time integral of the impedance changes was made by measuring the area under the impedance signal waveform recorded on chart paper. These experiments were performed at $14--18^{\circ} \mathrm{C}$.

Pharmacology. To study the effects of peptides and dopamine on the LHD or on its red and white hemiducts, the tissue was dissected free from the CNS, pinned to the bottom of the recording chamber, and attached to the movement transducer. Peptides were applied to the LHD by injecting a small bolus of drug directly into the line used to perfuse the duct through the genital artery, with one exception - the experiments in which we measured the concentration threshold for peptide action. In these experiments, the LHD was perfused continuously with a known concentration of the peptide. In experiments in which effects of the peptide were measured on the isolated red or white hemiduct of the LHD, the LHD was cut in half longitudinally, along the borders between the two hemiducts. This procedure disrupted the arterial supply to the tissue, so the isolated hemiducts were not perfused; rather, application of a bolus of peptide was made via the line used to superfuse the tissue. Peptide solutions were made fresh daily using the same batch of ASW that was used to bathe the preparation. R15 $\alpha 1$ peptide (Weiss et al., 1989) was purchased from Peninsula Laboratories (Belmont, CA) as "neuron-specific peptide." R15 $\beta 1$ peptide (Weiss et al., 1989) was obtained from the Howard Hughes Medical Institute's Protein Center Core Facility at Columbia University; dopamine was purchased from Sigma (St. Louis). Atrial gland extract (AGE) was prepared by the method described by Alevizos et al. (1991a). Bag cell extract (BCE) was prepared similarly, using a pair of bag cell clusters for each extract.

Data presentation and statistical analysis. All data are given as mean values \pm the standard error of mean (SEM). All statistical analyses of the results were made using a two-tailed $t$ test with a cutoff of $p=0.05$ for comparing the difference between means.

\section{Results}

\section{R15 innervates the large hermaphroditic duct}

Filling R 15's cell body with fluorescent dye and tracing its processes in the periphery revealed a rich innervation of the pericardium and the mesentery of the hepatopancreas, as described by Rittenhouse and Price (1985). In addition, we observed a previously undescribed site of innervation by R15, the LHD. Varicosities could be seen in all innervated regions (Fig. 1), suggesting that R15 may release its peptides at these sites.

Spontaneous bursting of R15 elicited no apparent contraction or relaxation of the mesentery of the hepatopancreas or the pericardium, even when using the nondesensitizing washing protocol that revealed the central effects of R15. Application of the $\mathrm{R} 15 \alpha 1$ peptide also was without effect. These may be sites where $\mathrm{R} 15$ releases its peptides into the general circulation, as suggested by Rittenhouse and Price (1985). In contrast, R 15 activity elicited strong contractions of the LHD.

\section{R15 activity causes contraction of the LHD}

Using the nondesensitizing washing protocol described above (see Materials and Methods), we recorded robust peristaltic movements of the LHD in response to spontaneous bursting activity in R15. The excitatory effect of R15 activity on the LHD was long lasting and was mimicked by application of the R $15 \alpha 1$ peptide. Both the peptide and R 15 produce anterograde peristaltic movements superimposed on a tonic contraction (Fig. $2 A-C$ ). The concentration threshold of the LHD response to $\mathrm{R} 15 \alpha \mathrm{l}$ peptide was about $3 \times 10^{-9} \mathrm{M}$ (data not shown).

To test if the effect on the LHD is mediated directly by R15, the saline bathing the abdominal ganglion was replaced with a modified saline in which $\mathrm{Ca}^{2+}$ was replaced with $10 \mathrm{~mm} \mathrm{Co}^{2+}$ to block synaptic transmission. The effect of $R 15$ bursting on the LHD persisted when synaptic transmission in the abdominal ganglion was blocked (Fig. $2 C$ ). In other experiments, the saline in the well was replaced by a high-divalent-cation saline to block polysynaptic pathways. The effect of R15 on the LHD also persisted in this high-divalent-cation solution $(N=2$; data not shown). These experiments do not exclude the possibility that R15 may activate peripheral neurons that mediate all or part of the response of the LHD. For example, catecholaminergic (presumably dopaminergic) neurons have been identified in the vaginal and the rostral parts of the LHD of Aplysia depilans and Aplysia fasciata (Salimova et al., 1987). We found that perfusion of the LHD with dopamine $\left(1-5 \times 10^{-5} \mathrm{M}\right)$ results in a prolonged relaxation of the organ, so it is unlikely that any peripheral effects of $\mathrm{R} 15$ arc mediated by these cells, unless thcy arc produccd by an unknown transmitter coreleased with dopamine. 

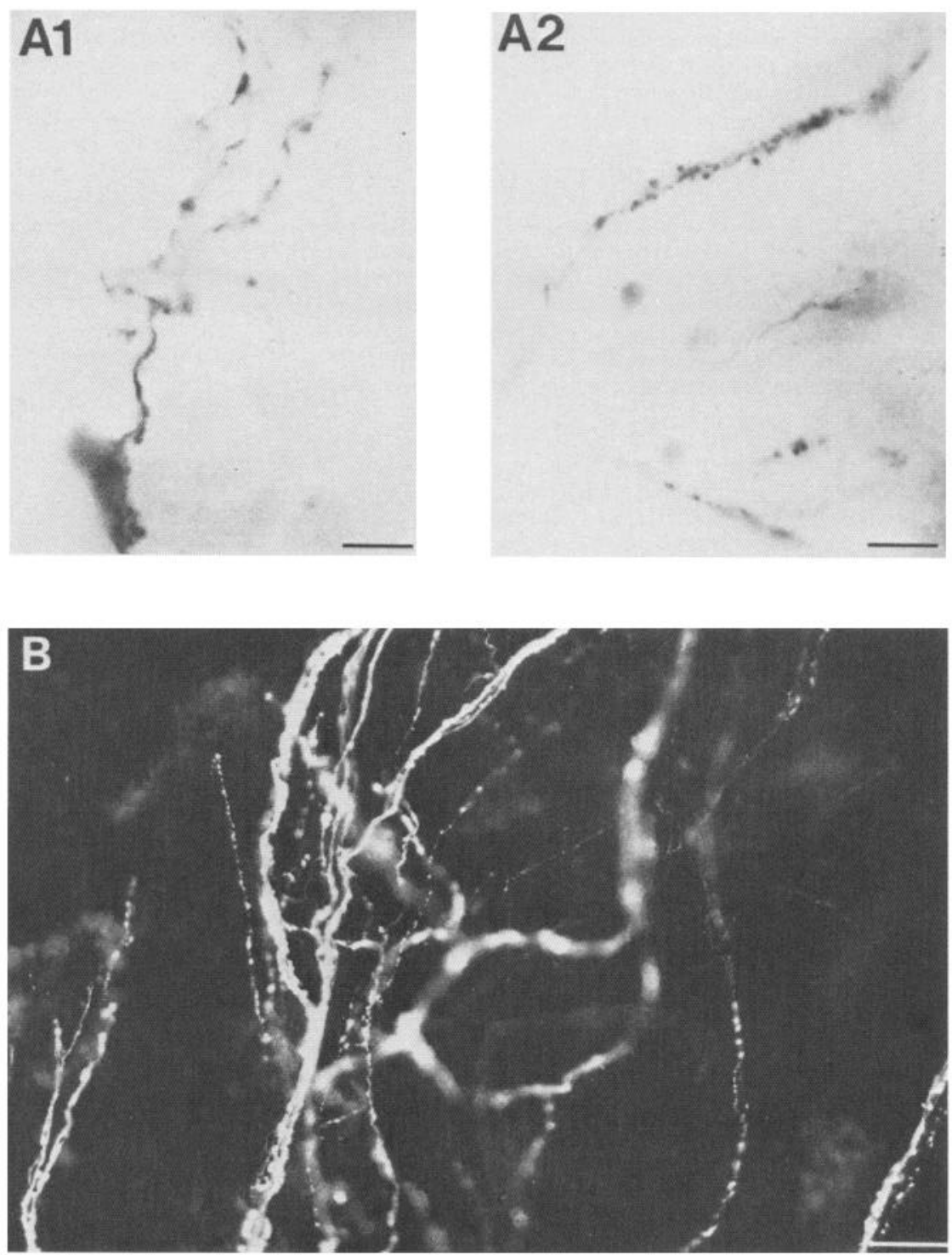

Figure 1. R15 innervates the LHD and the mesentery of the hepatopancreas: fluorescent micrographs of peripheral tissues made $48 \mathrm{hr}$ after injecting the soma of R15 with Lucifer yellow. $A 1$, $\mathrm{R} 15$ processes are seen in the LHD. $A 2$, Varicosities of R 15 are seen in the LHD. $B$, The mesentery of the hepatopancreas is richly innervated by R 15 processes. In control experiments, no autofluorescence was observed in these tissues. Scale bar, $160 \mu \mathrm{m}$ for $A$ and $B$.

\section{Physiological function of LHD contractions evoked by R15}

To determine the function of the contractions elicited in the LHD by R 15 activity, we attempted to localize the site of action of R15 to specific functional regions of the LHD. Because it is not possible to dissect the LHD without damaging its innervation, we applied R15 1 peptide, the putative transmitter of $\mathrm{R} 15$, in these experiments. The LHD consists of the white hemiduct, which transports sperm, the red hemiduct, which transports the egg cordon, and the atrial gland, an exocrine organ embedded in the wall of the red hemiduct that releases its peptide products into the lumen of the red hemiduct (Arch et al., 1980; Painter et al., 1985). R15 $\alpha 1$ peptide applied to isolated pieces of the red or the white hemiducts elicited contractions only of the red hemiduct. Higher concentrations of the peptide, up to $5 \times 10^{-6} \mathrm{M}$, had no effect on the white hemiduct (Fig. $2 D$ ). The R $15 \beta$ peptide had no excitatory or inhibitory effect on the contractile activity of the LHD (data not shown).

Successive periods of R15 bursting produced progressively smaller effects on the peristaltic activity of the LHD. In these experiments, R15 was released to burst for $15 \mathrm{~min}$ after a $2-\mathrm{hr}$ hyperpolarization period, and a $30 \mathrm{~min}$ period of silence was interpolated before the second 15-min period of bursting (Fig. $3 A$ ). Prolongation of the interval between two successive 10min periods of bursting in R15 resulted in complete recovery of the initial response with time (Fig. $3 B$ ). Successive applications of the $\mathrm{R} 15 \alpha 1$ peptide separated by a 30 -min interval also produced smaller and shorter-lasting contractions of the LHD, suggesting that the response to $\mathrm{R} 15 \alpha 1$ peptide may desensitize (Fig. 3C). Alternatively, the decremental response of the LHD to R15 and to its peptide may result from muscular fatigue.

In order to test if the responses to $\mathrm{R} 15$ and to $\mathrm{R} 15 \alpha 1$ peptide are mediated via the same receptor or postreceptor mechanism, a cross-desensitization experiment was performed. The effect of R15 was determined at the beginning of the experiment by releasing it from hyperpolarization for $5 \mathrm{~min}$. The $\mathrm{R} 15 \alpha 1$ peptide then was applied as a bolus until the LHD stopped responding to peptide application, at which time R15 was again released from hyperpolarization for $10 \mathrm{~min}$. The effect of $\mathrm{R} 15$ on the LHD was virtually eliminated during the second firing 
A

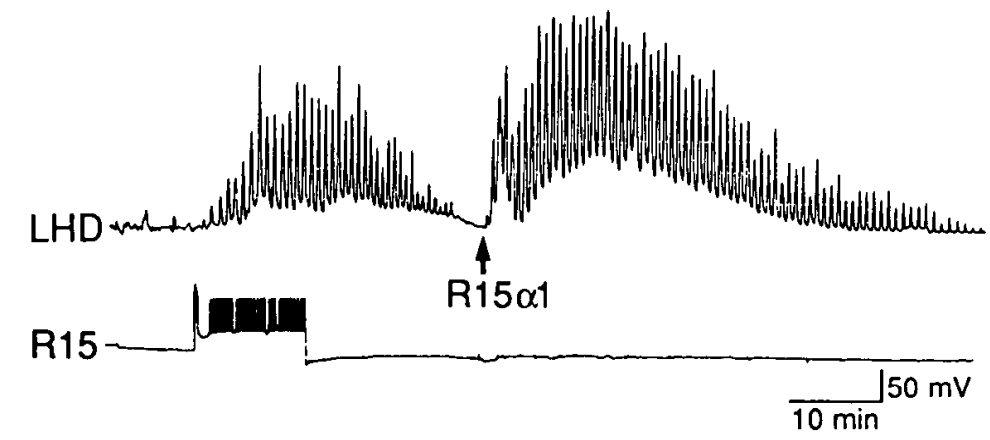

B
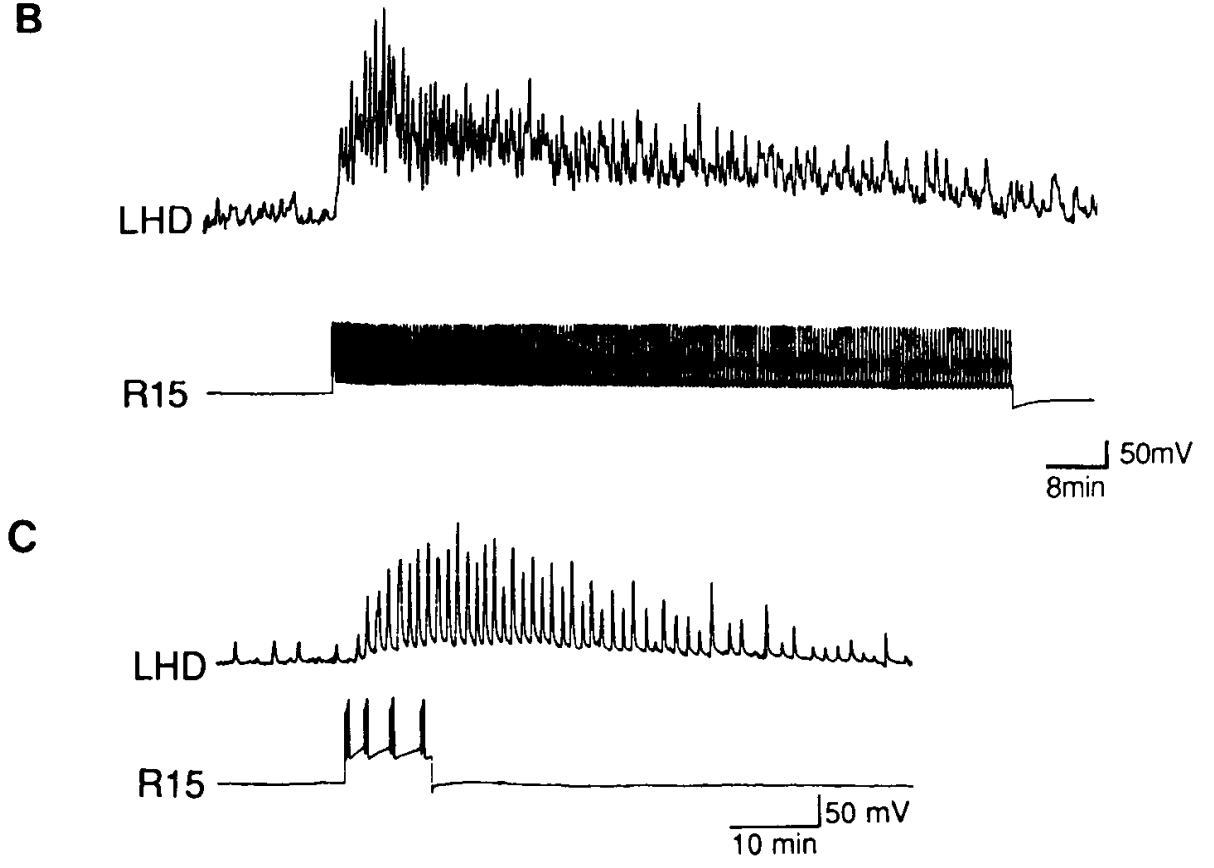

D
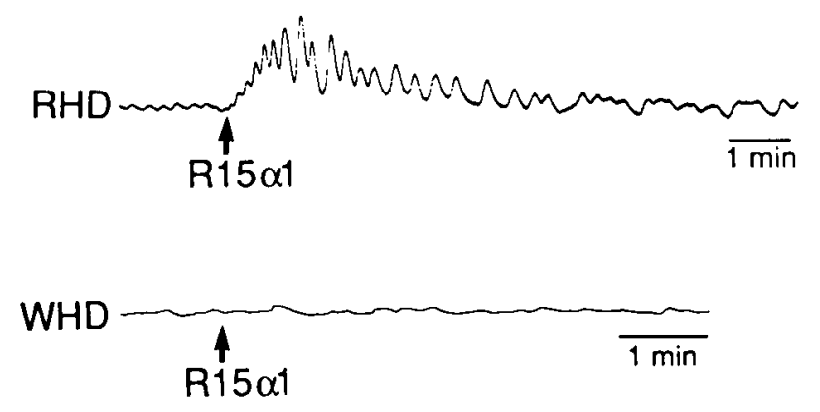

Figure 2. R15 and R15 $\alpha 1$ peptide elicit peristaltic activity of the LHD. $A, \mathrm{Al}-$ lowing R15 to burst spontaneously for $10 \mathrm{~min}$ after a $2-\mathrm{hr}$ hyperpolarization produces long-lasting peristaltic activity in the LHD. Application of R15 $\alpha 1$ peptide mimicks the effect of R15. A bolus of peptide $\left(0.1 \mathrm{ml}\right.$ of $\left.5 \times 10^{-6} \mathrm{M}\right)$ was injected into the perfusion line while the preparation was perfused continuously with ASW at a rate of $2.5-3 \mathrm{ml}$ $\min (N=6) . B$, The effect of R15 on the LHD wanes in time. Allowing R15 to burst spontaneously for $80 \mathrm{~min}$ elicits strong peristaltic activity in the LHD that decays in spite of the maintained bursting. $C$, The excitatory effect of $\mathrm{R} 15$ on the LHD persists when the isolated ganglion is bathed in a saline solution containing $10 \mathrm{mM} \mathrm{Co}^{2+}$ and $0 \mathrm{mM} \mathrm{Ca}^{2+}$ to block central synaptic transmission $(N=3) . D, \mathrm{R} 15 \alpha 1$ peptide initiates contractions exclusively in the red hemiduct of the LHD, the portion of the duct that propels the egg cordon. The preparation was superfused continuously with ASW (1-1.5 $\mathrm{ml} / \mathrm{min}$ ), and a 0.1 $\mathrm{ml}$ bolus of $5 \times 10^{-7} \mathrm{M}$ was injected into the superfusion line $(N=4)$. A 10 fold higher dose of $\mathrm{R} 15 \alpha 1$ peptide (a $0.1-\mathrm{ml}$ bolus of $5 \times 10^{-6} \mathrm{M}$ ) has no effect on the white hemiduct, the portion of the LHD that carrics the sperm $(N=$ 4).

period (Fig. $4 A$ ), at a time when the effect of R 15 activity on the LHD should be substantially recovered (Fig. $3 B$ ). Figure $4 B$ shows the mean of data from four such experiments. These data are consistent with the hypothesis that the $\mathrm{R} 15 \alpha 1$ peptide mediates the effects of R15 on the LHD. However, our data do not allow us to determine whether this inhibitory interaction between R 15 bursting and application of $R 15 \alpha 1$ peptide is due to muscle fatigue or to desensitization.

The fact that $\mathrm{R} 15$ bursting generates strong peristaltic activity of the LHD suggests that R15 may play a role in egg-laying behavior. However, such peristalsis has not been implicated previously in the transport of the mature egg cordon, which is assumed to be propelled by means of ciliary movements in the lumen of the red hemiduct (Thompson and Bebbington, 1969;
Coggeshall, 1972). To test if the LHD exhibits an enhanced peristaltic activity during egg laying, the movements of the LHD in intact animals were determined by measuring impedance changes with chronically implanted electrodes. None of the four animals used in these experiments laid eggs spontaneously, so egg laying was induced by injection of AGE (Arch et al., 1978). The injection of AGE induced characteristic ovipository behavior (Arch and Smock, 1977), and the egg cordon began to appear on the substrate an average of $29.6 \pm 4.4 \mathrm{~min}$ after injection, well within the range of previously reported latencies (Ferguson et al., 1989). Large, phasic movements of the LHD, which gradually decayed over the course of $1 \mathrm{hr}$, occurred synchronously with the anterograde movement of the eggs (Fig. 5). The egg cordon was mostly mucoid in appearance and relatively 
A

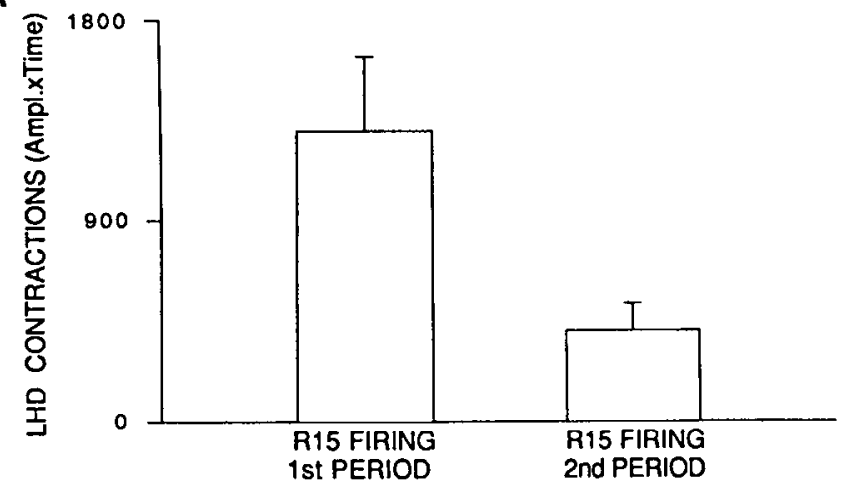

B

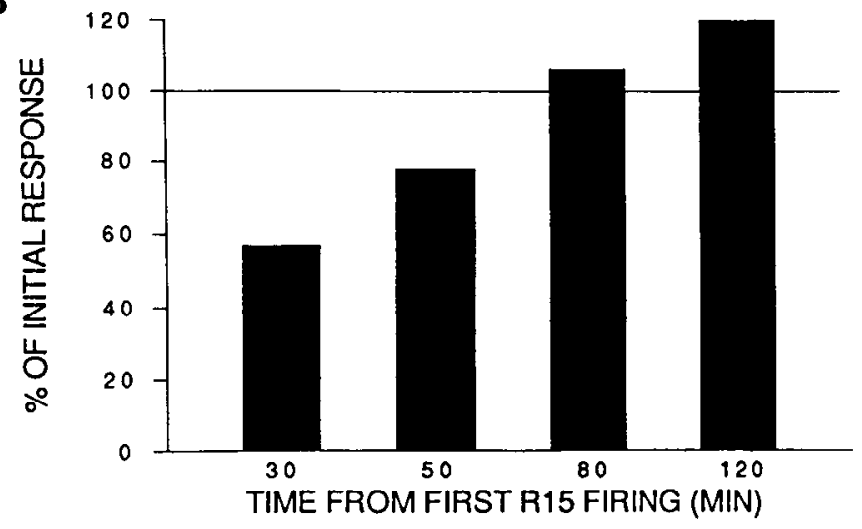

C

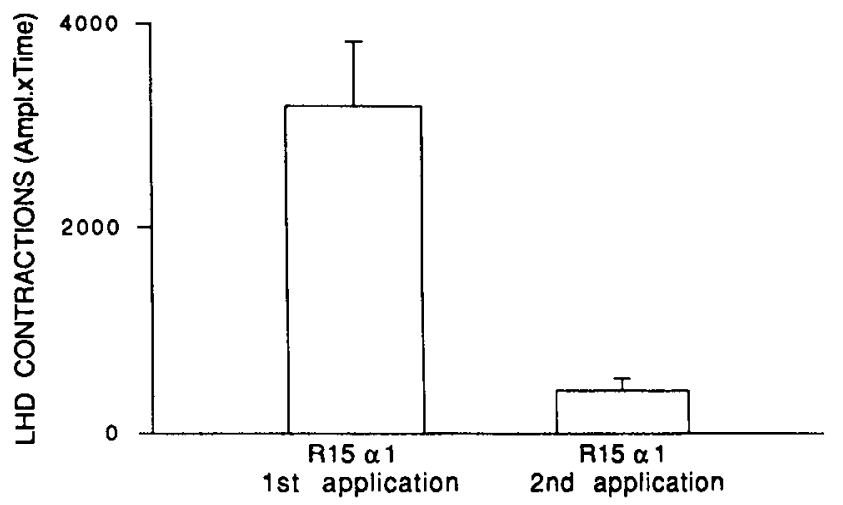

Figure 3. The excitatory effects of R 15 and the R $15 \alpha 1$ peptide on the LHD decrease with repetition. $A, \mathrm{R} 15$ was allowed to burst spontaneously for $15 \mathrm{~min}$ after $2 \mathrm{hr}$ of hyperpolarization. R15's firing elicited peristaltic activity in the LHD that decayed over a period of $40 \mathrm{~min}$ from the start of firing. Then R15 was released for a second 15-min period. The effect on the LHD was significantly smaller during this second period of R15 firing $(N=4)$. (The number of spikes in R15 increased during the second firing period compared to the first by $11.4 \%$.) Error bars represent SEM. B, The recovery of R15's excitatory effect on the LHD requires a long-lasting hyperpolarization of $\mathrm{R} 15$. R 15 was released from a 2-hr hyperpolarization period and allowed to burst for $10 \mathrm{~min}$, eliciting peristaltic activity of the LHD. In each of the four preparations, R15 was also released from hyperpolarization a second time, after a variable time interval (indicated under bars). Prolongation of the interval between the first and the second firing periods allows the excitatory effect of R15 to recover to its initial value. $C$, The effect of the second of two successive applications of R15 $\alpha 1$ peptide to the LHD is significantly smaller than that of the first $\left(0.1-\mathrm{ml}\right.$ bolus of $5 \times 10^{-6}$ M peptide; $N=4$ ). Error bars represent SEM.
A
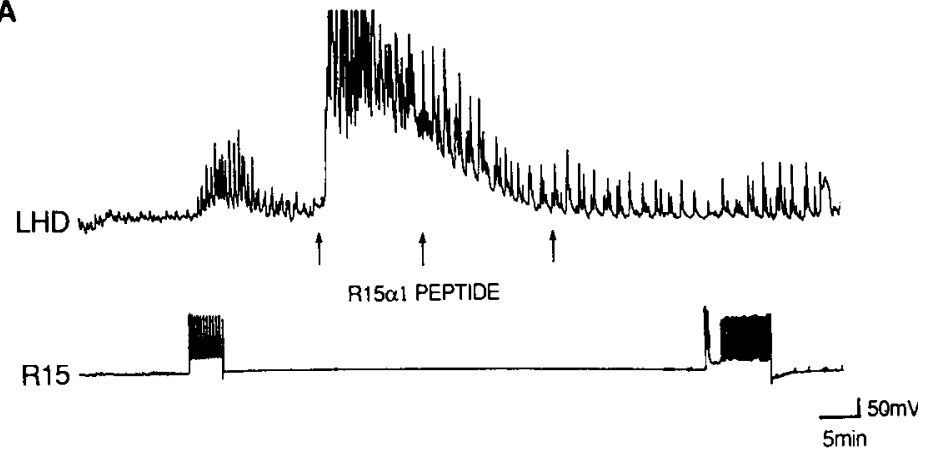

B

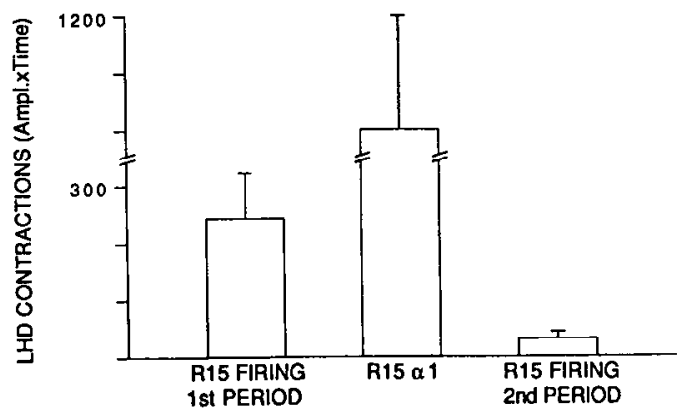

Figure 4. Multiple applications of the R15 1 peptide block the effect of $\mathrm{R} 15$ on the LHD. $A, \mathrm{R} 15$ was allowed to burst spontaneously for 5 min after a 2-hr hyperpolarization, in order to test the responsiveness of the LHD to R15. Then, three successive applications of the R $15 \alpha 1$ peptide $\left(0.1-\mathrm{ml}\right.$ bolus of $\left.5 \times 10^{-6} \mathrm{M}\right)$ were administered before $\mathrm{R} 15$ was released from hyperpolarization for $10 \mathrm{~min}$. The blocking action of the peptide may be due to cross-desensitization between the peptide and R15, though the possibility of muscular fatigue cannot be excluded. $B$, Group data from the experimental protocol in $A$. The excitatory effect caused by the second period of R15 bursting was significantly smaller than that caused by the first $(N=4)$. The middle bar represents the sum of the contractions produced by the three applications of peptide. Measurements were made up to $10 \mathrm{~min}$ after the last peptide application. Error bars represent SEM.

short $(6.1 \pm 3.5 \mathrm{~cm})$ as compared to a spontaneously laid egg cordon, which is gelatinous in nature and can reach several tens of centimeters in length in animals of this size. These differences may result either because trauma is produced in the LHD by the implantation of the wire, or because bag-cell bursts elicited by AGE injection may be less intense or shorter in duration than the naturally occurring bursts that trigger spontaneous egg laying. The possibility that the impedance changes during egg laying are due to direct actions of AGE and bag cell peptides on the LHD can be ruled out on the basis of two observations: AGE and BCE do not trigger peristaltic activity when perfused through the LHD in vitro ( $N=3$ for each condition; data not shown), and bag cell discharge does not produce peristaltic activity of the LHD if R15 is kept hyperpolarized (Fig. 6). However, the data do not show conclusively that the effect of AGE on peristalsis is caused by a bag cell discharge exciting R15, which in turn acts on the LHD. Part of the effect of AGE could be mediated by a direct effect on R15, caused by the ELH-like peptides found in the atrial gland (Nagle et al., 1986; Rothman et al., 1986). But there is abundant evidence that the bag cells excite R15 (Branton et al., 1978), so the data are at least consistent with the hypothesis that peristaltic activity of the LHD 
A1

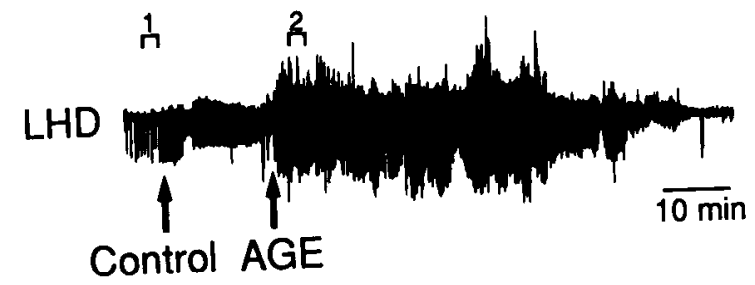

A2
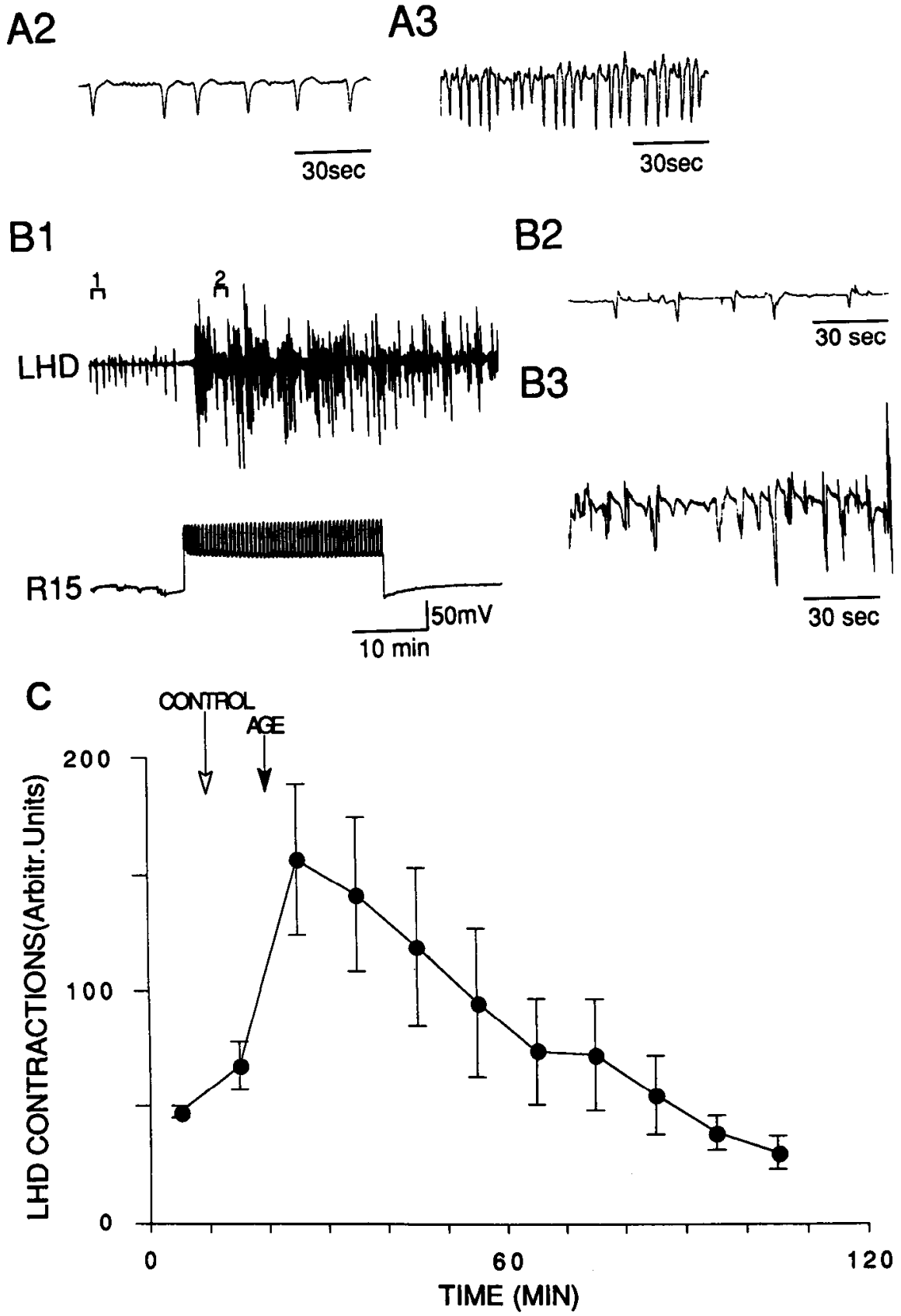

Figure 5. The LHD contracts during egg laying. $A$, The movements of the LHD of intact animals were monitored by measurements of impedance changes of the organ. A1, Injection of AGE into the hemocoel of the animal caused vigorous activity in the LHD. AGE injection consisted of $20 \%$ of a homogenized atrial gland in $0.2 \mathrm{ml}$ of ASW. The control injection consisted of an equivalent volume of ASW. A2, A high-speed record of the baseline activity of the organ is shown (from bracket 1 in $A 1$ ). $A 3$, The high speed record from bracket 2 of the record in $A 1$ shows that the contractions are more frequent and larger in amplitude after the AGE injection. $B 1$, The activity of the LHD in response to $R 15$ firing as recorded by impedance measurements in the semi-intact preparation. R 15 was allowed to burst spontaneously for 25 min after a 2-hr hyperpolarization period. $B 2$, The activity of the LHD during the base line period before R 15 firing (from bracket $I$ in $B I$ ). $B 3$, The activity of the LHD during the R15 firing period (from bracket 2 in $B I)$. $C$, Average of four experiments such as illustrated in $A 1-3$, showing LHD contractions during egg laying. Injection of AGE into animals produccs vigorous activity of the LHD that decayed over a period of $60 \mathrm{~min}$, as monitored by impedance change measurements. Error bars represent SEM.

is a functional component of oviposition that is elicited by bag cell activation of $\mathrm{R} 15$.

Our experiments also address the question of how respiratory pumping could be activated by a bag-cell burst. Schaefer and Brownell (1986) reported that triggering a bag-cell burst in vitro by electrical stimulation of the bag cells elicits an increase in the frequency of respiratory pumping. More recently, Alevizos et al. (1991a, Fig. 13) provided evidence that an excitatory effect of the bag cells on the R25/L25 network, which drives respiratory pumping, is mediated by excitation of $\mathrm{R} 15$. The raw data in Figure 7 corroborate their observation that when the bag cells fire in an AGE-elicited burst, any effect they may have on respiratory pumping is blocked if R15 is prevented from firing. In all four experiments in which AGE was injected into intact animals, visual observation confirmed that the injection caused an increase in the rate of respiratory pumping, which lasted 

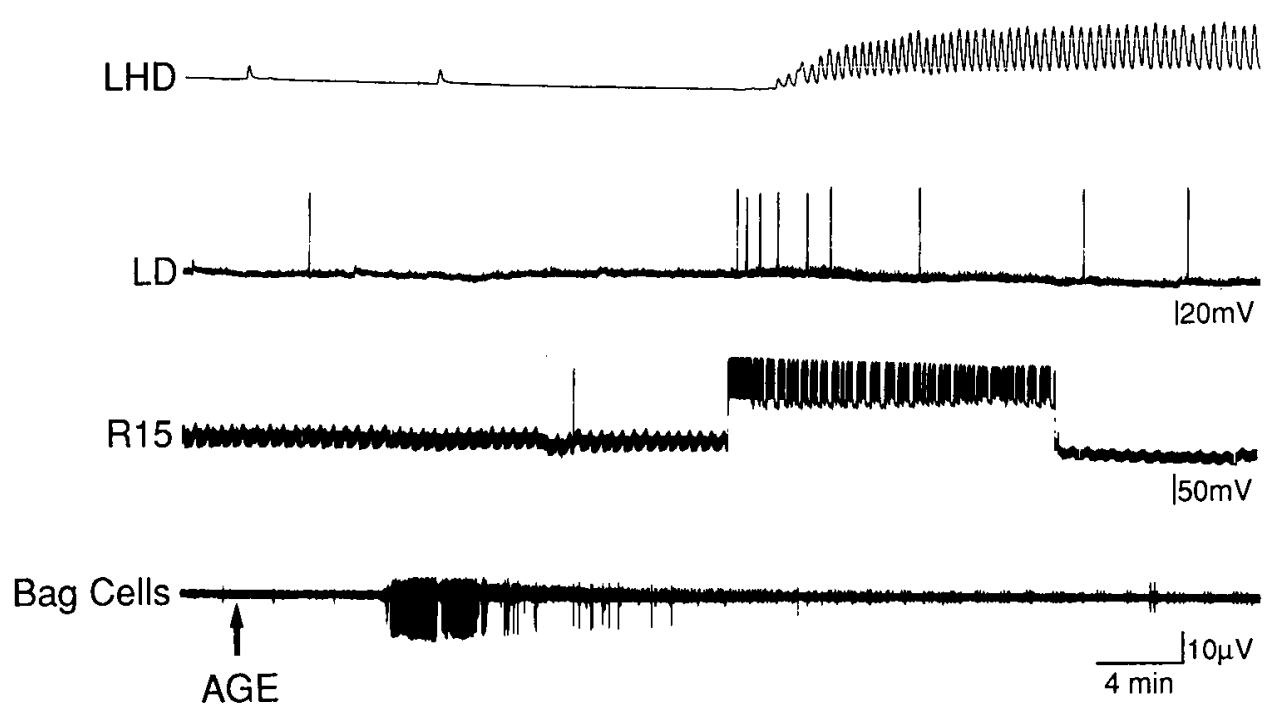

Figure 6. R15 activity is necessary for LHD contractions and modulation of respiratory pumping rate during AGE-induced bag cell discharge. The abdominal ganglion was kept connected by the pleuroabdominal connectives to the head ganglia, which were isolated from the rest of the nervous system in a subchamber. R15 was hyperpolarized for $2 \mathrm{hr}$ before the bag cells were excited. An LD cell was used to monitor activity in the R25/L25 network, and peristaltic activity of the LHD was recorded by means of a displacement transducer. Activity in bag cells was recorded with an extracellular electrode, positioned above the left bag cell cluster. Application of AGE to the head ganglia (1/8 of an homogenized atrial gland) caused the bag cells to discharge. The activity of the LHD and the rate of R25/L25 cell bursting did not change for 15 min after the bag cell discharge. At that point, releasing R15 from hyperpolarization and allowing it to burst spontaneously for 15 min elicited vigorous peristaltic activity of the LHD and an increase in the rate of respiratory pumping $(N=2)$.

approximately $20-30 \mathrm{~min}$ and consisted mostly of weak bursting. However, application of AGE on the isolated, deshcathed abdominal ganglion produced a similar effect, so it is not possible to say if the effect in vivo is due to direct action of the AGE on the R25/L25 cells, or if it is mediated through another neural component such as R15.

\section{Discussion}

The possible role of $R 15$ in egg laying

The demonstration that R15 has a large terminal field in the LHD suggests that it may modulate the contractile activity of this structure during egg laying. Four observations further support this hypothesis: (1) The bag cells trigger egg laying when they burst in vivo (Pinsker and Dudek, 1977). (2) R15 is excited by the bag cells in vitro (Branton et al., 1978). (3) R15 activity increases anterograde peristalsis only in the red hemiduct of the LHD, which serves as the pathway by which the eggs leave the body; it has no effect on the white hemiduct, which mediates the transfer of sperm. (4) Similar peristaltic activity of the LHD occurs during AGE-induced egg laying in the intact animal.

The R15-induced contractile activity of the LHD may be a crucial link between the bag-cell burst and oviposition. ELH released by the bag cells elicits release of oocytes from the ovotestis to the opening of the small hermaphroditic duct (Coggeshall, 1970). In the small hermaphroditic duct and the accessory genital mass, the eggs are propelled by ciliary action to the opening of the LHD (Coggeshall, 1972). In the LHD, the mature egg cordon is formed and obtains its adhesive coating. The muscular contractions may aid in distributing the secretions of the hermaphroditic duct evenly over the egg cordon. Moreover, the propulsion of the thick, sticky cordon may require, in addition to the ciliary movement, a force imparted by the peristaltic movements of the LHD. Perfusion of the LHD with BCE does not directly elicit peristaltic activity, so the activation of R15 appcars to be cssential for triggering the peristalsis that contributes to egg laying.

The data obtained from this study suggest that $\mathrm{R} 15$ may have a crucial function in the control of egg laying. In addition to its effect on the LHD, the activation of R15 by the bag cell burst also appears to have at least 2 additional effects: activation of the R25/L25 network that triggers respiratory pumping (Schaefer and Brownell, 1986; Alevizos et al., 1991a) and contraction of the pleuroabdominal connectives, mediated by excitation of L7 (Alevizos et al., 1991b). Inducing the bag cells to fire by applying AGE to the head ganglia does not modulate the rate of discharge of the R25/L25 network or the peristaltic activity of the LHD as long as R 15 is kept hyperpolarized; nor does bag cell discharge per se affect L7 (A. Alevizos, unpublished observations). However, release of R15 from hyperpolarization 15 min after the start of a bag cell discharge initiates bursting of the R25/L25 cells and peristalsis of the LHD (Fig. 6). Thus, $R 15$ could play a critical role in integrating various components of neural activity during oviposition, but for one complication: all three of R15's synaptic actions decrement rapidly, and R15 is normally endogenously active in vitro. Results from chronic recording experiments eliminate this reservation, however, because they indicate that R 15 is normally silent in vivo (Alevizos et al., 1991a). It appears to be a conditional burster that presumably bursts during egg laying.

The results of this study and those of other investigators give rise to the following hypothesis (Fig. 7): During bag cell discharge, the silent R15 is excited by the release of ELH (Branton et al., 1978) and starts to burst, causing the release of R15's peptides in the CNS and the periphery. Cells in the R25/L25 network are excited and increase their rate of bursting discharges (Alevizos et al., 1991a), producing a high rate of respiratory 


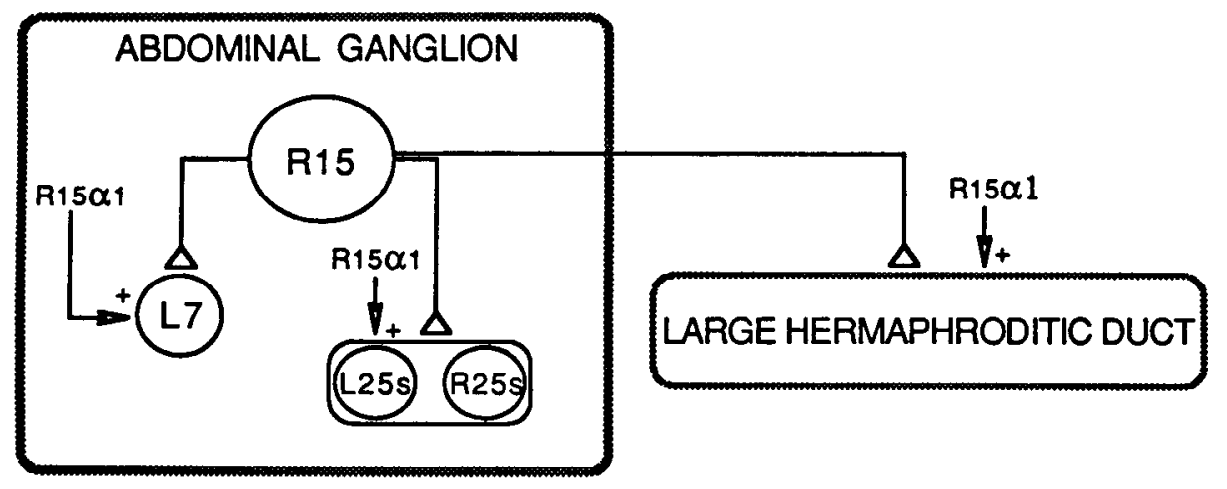

Figure 7. Schematic diagram of the known synaptic connections made by $\mathrm{R} 15$ in the CNS and the periphery, and the actions of the $R 15 \alpha 1$ peptide on the R15 targets. All of the synaptic and pharmacological effects shown in this diagram persisted in solutions that block polysynaptic pathways. Triangles represent chemical excitatory connections. Arrows indicate the excitatory effects of the $\mathrm{R} 15 \alpha 1$ peptide.

pumping, which helps the animal meet the enhanced demands for oxygen uptake during oviposition. In addition, the pressure surge that accompanies the gill contraction during respiratory pumping may help to promote the systemic circulation of ELH (Kanz and Quast, 1990). L7 is also excited by R15, causing a contraction of the muscular sheath of the connectives, which may result in an increase in its vascular resistance (Alevizos et al., 1991b). Such an effect would reduce the rate at which bag cell peptides are washed out of the cuff regions of the connectives, thus enhancing their role in mediating mutual excitation of the bag cell processes. Alternatively, the contraction of the connectives may serve as a postural adjustment (Umitsu et al., 1987). ELH released by the bag cells eventually reaches the ovotestis, where it acts on the follicles of the ovotestis to release the mature oocytes (Coggeshall, 1970). They then pass via ciliary motion to the glands of the accessory genital mass, to be fertilized and to receive the secretions that will form the coating of the egg cordon (Coggeshall, 1972). Release of R15 $\alpha$ l peptide into the general circulation also promotes the water uptake that is necessary for the albumen and mucus glands to form their secretory products. The ova pass from the accessory genital mass to the red hemiduct of the LHD, where they obtain their adhesive coating. The mature egg cordon is propelled to the genital groove by the strong peristaltic movements of the red hemiduct that are directly elicited by the activity of R15. The egg cordon is moved by ciliary action to the rostral end of the genital groove, at which point it is deposited on the substrate. A critical test of this hypothesis will be to determine whether R 15 is excited by the bag cells during spontaneous egg laying in vivo.

If $\mathrm{R} 15$ does contribute to egg laying, it may also participate in other behaviors. R15 has widespread effector fields, as do identified cells L7 (Alevizos et al., 1989a) and L10 (Koester and Alevizos, 1989) of the abdominal ganglion and the giant cerebral neuron of the cerebral ganglion (Weiss and Kupfermann, 1976). Perhaps the diverse outputs of these neurons are gated by various mechanisms, such as heterosynaptic facilitation or depression, or by postsynaptic modulation of their transmitter actions, so that their synaptic effects are selectively expressed during different behavioral programs.

\section{R15a1 peptide: possible role in initiation and termination of egg-laying behavior}

Evidence presented in the two preceding papers suggests that $\mathrm{R} 15 \alpha 1$ peptide mediates the excitatory actions of R15 on the R25/L25 network and on L7. A key observation in support of this hypothesis is the apparent cross-desensitization between the physiological actions of R15 and the pharmacological actions of $\mathrm{R} 15 \alpha 1$ peptide. The $\mathrm{R} 15 \alpha \mathrm{l}$ peptide also may mediate the excitatory effects of R15 on the LHD. As with L7 and the $\mathrm{R} 25 / \mathrm{L} 25$ cells, the actions of R15 and of the R15 1 peptide on the LHD appear to cross-desensitize. However, because we recorded only contractile activity, we cannot rule out the possibility that muscle fatigue or refractoriness accounts for some or all of the interference between the effects of R15 and R15 1 peptide on the LHD. Our current working hypothesis is that $\mathrm{R} 15 \alpha 1$ peptide mediates the effect of $\mathrm{R} 15$ on the R25/L25 cells, L7, and the LHD and that these actions desensitize at physiological firing frequencies, accounting for the decay in the response of all three postsynaptic followers to maintained firing of $\mathrm{R} 15$.

If $\mathrm{R} 15$ bursts during egg laying, desensitization of the $\mathrm{R} 15 \alpha 1$ receptors are likely to have two important functional effects during egg laying: (1) The different time courses of the apparent desensitization produced by the $R 15 \alpha 1$ peptide released by $R 15$ may determine the time course of R15's synaptic effects. For example, the effect of maintained R15 firing on the isolated LHD decays over approximately 60-80 min, which is about the time required for the egg cordon to be deposited. On the other hand, the effects of maintained bursting of R15 on the R25/L25 network and on L7, which decay more rapidly (Alevizos et al., $1991 \mathrm{a}, \mathrm{b})$, may be related to adjustments required at the start of egg laying, during the initial phase of bag cell discharge (Kaczmarek and Kauer, 1983). Thus, the different rates of desensitization at different tissues may be important in determining the timing of the various components of this complex behavioral pattern. (2) The long-lasting desensitization of R15's synaptic actions may complement the long-lasting refractoriness of the bag cells that follows a population burst (Kaczmarek and Kauer, 1983; Brown and Mayeri, 1989), with both phenomena combining to ensure that egg laying occurs in discrete episodes. It will be necessary to determine the firing pattern of $\mathrm{R} 15$ during egg laying in the intact animal to determine the functional significance of the activation and desensitization of its synaptic actions.

\section{References}

Alevizos A, Bailey CH, Chen M, Koester J (1989a) Innervation of vascular and cardiac muscle of Aplysia by multimodal motoneuron L7. J Neurophysiol 61:1053-1063.

Alevizos A, Weiss KR, Koester J (1989b) R15 activates respiratory pumping, motoneuron L7, and the hermaphroditic duct in Aplysia. Soc Neurosci Abstr 15:1140. 
Alevizos A, Weiss KR, Koester J (1991a) Synaptic actions of identified peptidergic neuron R15 in Aplysia. I. Activation of respiratory pumping. J Neurosci 1 1:1263-1274.

Alevizos A, Weiss KR, Koester J (1991b) Synaptic actions of identified peptidergic neuron R15 in Aplysia. II. Contraction of pleuroabdominal connectives mediated by motoneuron L7. J Neurosci 11:12751281.

Arch S (1976) Neuroendocrine regulation of egg laying in Aplysia californica. Am Zool 16:167-175.

Arch S, Smock T (1977) Egg-laying behavior in Aplysia californica. Behav Biol 19:45-54.

Arch S, Smock T, Gurvis R, McCarthy C (1978) Atrial gland induction of the egg-laying response in Aplysia californica. J Comp Physiol 128: 67-70.

Arch S, Lupatkin J, Smock T, Beard M (1980) Evidence for an exocrine function of the Aplysia atrial gland. J Comp Physiol 141:131-137.

Branton WD, Arch S, Smock T, Mayeri E (1978) Evidence for mediation of a neuronal interaction by a behaviorally active peptide. Proc Natl Acad Sci USA 75:5732-5736.

Brown RO, Mayeri E (1989) Positive feedback by autoexcitatory neuropeptides in neuroendocrine bag cells of Aplysia. J Neurosci 9:14431451 .

Coggeshall RE (1970) A cytologic analysis of the bag cell control of egg laying in Aplysia. J Morphol 4:461-486.

Coggeshall RE (1972) The structure of the accessory genital mass in Aplysia californica. Tissue Cell 4:105-127.

DiVirgilio F, Steinberg TH, Swanson JA, Silverstein SC (1988) Fura-2 secretion and sequestration in macrophages. J Immunol 140: 915-920.

Ferguson GP, Ter Maat A, Parsons DW, Pinsker HM (1989) Egg laying in Aplysia: I. Behavioral patterns and muscle activity of freely behaving animals after selectively elicited bag cell discharges. J Comp Physiol A 164:835-847.

Kaczmarek LK, Kauer JA (1983) Calcium entry causes a prolonged refractory period in peptidergic neurons of Aplysia. J Neurosci 3: 2230-2239.

Kandel ER (1976) The cellular basis of behavior. San Francisco: Freeman.

Kanz JE, Quast WD (1990) Respiratory pumping seizure: a newly discovered spontaneous stereotyped behavior pattern in the opisthobranch mollusc Aplysia californica. J Comp Physiol A 166:619-627.

Koester J, Alevizos A (1989) Innervation of the kidney of Aplysia by L10, the LUQ cells, and an identified peripheral motoneuron. J Neurosci 9:4078-4088.
Nagle GT, Painter SD, Blankenship JE, Dixon JD, Kurosky A (1986) Evidence for the expression of three genes encoding homologous atrial gland peptides that cause egg laying in Aplysia. J Biol Chem 261: 7853-7859.

Painter SD, Kalman VK, Nagle GT, Zuckerman RA, Blankenship JE (1985) The anatomy and functional morphology of the large hermaphroditic duct of species of Aplysia, with special reference to the atrial gland. J Morphol 186:167-194.

Pinsker MH, Dudek FE (1977) Bag cell control of egg laying in freely behaving Aplysia. Science 197:490-493.

Rao G, Barnes CA, McNaughton BL (1986) Intracellular fluorescent staining with carboxyfluorescein: a rapid and reliable method for quantifying dye-coupling in mammalian central nervous system. J Neurosci Meth 16:251-263.

Rittenhouse AR, Price CH (1985) Peripheral axons of the parabolic burster neuron R15. Brain Res 333:330-335.

Rothman BS, Weir G, Dudek FE (1983) Egg-laying hormone: direct action on the ovotestis of Aplysia. Gen Comp Endocrinol 52:134 141.

Rothman BS, Hawke DH, Brown RO, Lec TD, Dehghan AA, Shively JE, Mayeri E (1986) Isolation and primary structure of the califins, three biologically active egg-laying hormone-like peptides from the atrial gland of Aplysia californica. J Biol Chem 261:1616-1623.

Salimova NB, Sakharov DA, Milosevic I, Rakic L (1987) Catecholamine-containing neurons in the peripheral nervous system of $A p l y$ sia. Acta Biol Hung 38:203-212.

Schaefer M, Brownell PH (1986) Modulation of a respiratory motor program by peptide-secreting neurons in Aplysia. J Neurobiol 17: 121-126.

Thompson TE, Bebbington A (1969) Structure and function of the reproductive organs of three species of Aplysia (Gastropoda: Opisthobranchia). Malacologia 7:347-380.

Umitsu Y, Matsumoto H, Koike H (1987) Active contraction of nerve bundle and identification of a nerve-contractor motoncuron in Aplysia. J Neurophysiol 58:1016-1034.

Weiss KR, Kupfermann I (1976) Homology of the giant serotonergic neurons (metacerebral cells) in Aplysia and pulmonate molluscs. Brain Res 117:33-49.

Weiss KR, Bayley H, Lloyd PE, Tenenbaum R, Gawinowicz Kolks MA, Buck L, Cropper EC, Rosen SC, Kupfermann I (1989) Purification and sequencing of neuropeptides contained in neuron R15 of Aplysia californica. Proc Natl Acad Sci USA 86:2913-2917. 